\title{
Topology invariance in Percolation Thresholds
}

\author{
Serge Galam and Alain Mauger \\ Laboratoire des Milieux Désordonnés et Hétérogènes * \\ Tour 13 - Case 86, 4 place Jussieu, \\ 75252 Paris Cedex 05, France
}

(june 3, 97)

PA Classification Numbers: 64.60 A, 64.60 C, 64.70 P

\begin{abstract}
An universal invariant for site and bond percolation thresholds $\left(p_{c s}\right.$ and $p_{c b}$ respectively) is proposed. The invariant writes $\left\{p_{c s}\right\}^{\frac{1}{a_{s}}}\left\{p_{c b}\right\}^{-\frac{1}{a_{b}}}=$ $\delta / d$ where $a_{s}, a_{b}$ and $\delta$ are positive constants, and $d$ the space dimension. It is independent of the coordination number, thus exhibiting a topology invariance at any $d$. The formula is checked against a large class of percolation problems, including percolation in non-Bravais lattices and in aperiodic lattices as well as rigid percolation. The invariant is satisfied within a relative error of $\pm 5 \%$ for all the twenty lattices of our sample at $d=2, d=3$, plus all hypercubes up to $d=6$.
\end{abstract}

\footnotetext{
*Laboratoire de l'Université P. et M. Curie - Paris 6, associé au CNRS (URA n ${ }^{\text {800) }}$
} 


\section{Introduction}

Percolation phenomena are active in a rather broad spectrum of physical and non-physical problems [1]. It is now a full part of Statistical Physics. However, most studies have been devoted to percolation on regular lattices, merely for convenience [2]. But even for these lattices, exact results are scarce. In particular site and bond percolation thresholds are known exactly only at $d=2$ and for a few cases. Otherwise all available thresholds are given by numerical estimates [1].

In the past several attempts were made to unify percolation thresholds. None was really satisfactory. we have presented very recently an universal power law to yield both site and bond percolation thresholds, $p_{c s}$ and $p_{c b}$ respectively, within an excellent accuracy [3],

$$
p_{c s}=p_{0 s}\{(d-1)(q-1)\}^{-a_{s}},
$$

for site and

$$
p_{c b}=p_{0 b}\left\{\frac{(d-1)(q-1)}{d}\right\}^{-a_{b}}
$$

for bond with $d$ the space dimension and $q$ the coordination number. The formula yields thresholds for any Bravais lattice, at any dimension with an impressive accuracy [3].

For $d \leq 6$, two different classes were found, and identified by distinct parameter sets $\left\{p_{0 i} ; a_{i}\right\}$ where $i=s, b$. Both Eqs (1) and (2) are satisfied within few per cent for all the lattices inside a given class. The first class includes two-dimensional triangle, square and honeycomb lattices. It is characterized by $\left\{p_{0 s}=0.8889 ; a_{s}=0.3601\right\}$ for site dilution and by $\left\{p_{0 b}=0.6558 ; a_{b}=\right.$ $0.6897\}$ for bond dilution. Two-dimensional Kagomé and all other lattices of cubic symetry (for $3 \leq d \leq 6$ ) constitute the second class which is characterized by $\left\{p_{0 s}=1.2868 ; a_{s}=0.6160\right\}$ and $\left\{p_{0 b}=0.7541 ; a_{b}=0.9346\right\}$ for sites and bonds respectively.

Above results were obtained using a sample which includes most common lattices mentioned above [3]. This is, however, quite restrictive, because of the new trends in modern percolation problems related in particular to directed 
percolation, percolation in aperiodic lattices, and rigidity percolation.

In the present work, we use a much broader lattice sample including percolation in non-Bravais lattices and in aperiodic lattices, and rigidity percolation as well. However, extension of Eqs. (1,2) to non regular lattices is questionable, in particular with respect to the use of the coordination number $q$ as a relevant parameter in our universal formula.

Indeed some lattices with equal $d$ and $q$ have different $p_{c}$, like, for instance, the stacked triangle lattice and $b c c$ lattices at $d=3[4,5]$. In this paper we name stacked triangle the 3d-lattice also called hexagonal lattice in the litterature, to avoid any confusion with hexagonal compact lattice, and the 2d-triangle lattice also called hexagonal by some authors. Moreover Eqs. $(4,5)$ are found not to hold for many non-regular lattices, which is indeed not surprising. Percolation should depend on the degree of lattice anisotropy, which is not included in Eqs. $(1,2)$ where only the bare coordination number $q$ appears.

Therefore, at this stage, with respect to our power law, there is no proof that, besides the dimension $d$, there exists one single parameter which contains all the relevant information on the lattice topology. Yet, this parameter entering Eqs $(1,2)$, if it exists, clearly cannot be reduced to the coordination number $q$.

Nevertheless, it is worth noting that for each lattice, there exists one single parameter $q_{\text {eff }}$ which reproduces, within an excellent accuracy, both percolation thesholds from Eqs. $(4,5)[6]$. However, at present, we have no scheme to calculate $q_{e f f}$, which then, has to be determined from the values of site or bond percolation thresholds.

To bypass this difficulty, we can eliminate $q$ from Eqs. $(1,2)$ by combining them to obtain, for the first time in percolation theory, a universal invariant which combines $p_{c s}, p_{c b}$ and $d$. The formula exhibits a topology invariance and holds for all percolation problems, including percolation in non-Bravais lattices and in aperiodic lattices as well as rigidity percolation. The invariant is satisfied, at worst within $\mp 0.05$ for all the twenty lattices of our sample at $d=2, d=3$, plus the hypercubes up to $d=6$ with the same relative accuracy.

\section{The Invariant}

\section{A. Non-regular lattices}


Let us first review the large spectum of non-regular lattices we include in our sample with their main characteristics. The stacked triangle lattice is anisotropic with 6 equivalent nearest neighbors $(n n)$ in the $a, b$ plane (bonding angle is $60^{\circ}$ ), and two non equivalent neighbors along the $c$ axis perpendicular to it (bonding angle is $90^{\circ}$. $p_{c s}, p_{c b}$ for this lattice have been determined very recently [4]. On another hand, the hexagonal close packed (hcp) lattice is a non-Bravais lattice, with two lattice sites per unit cell. The percolation thresholds of the hcp lattice have been determined a long time ago [7].

Aperiodic lattices are represented by quasicrystals. Besides their own interest, these aperiodic materials with long range order can serve as models for alloy materials, hence a growing interest in their percolation thresholds. Our sample includes Penrose tiling [8-10], octagonal and dodecagonal tilings [11] with chemical links (connection via the tile edges), and ferromagnetic links (connection through the diagonal of the tiles, which are shorter than the tile edges) [12].

The sample is also enriched by the dual of the lattices. Percolation thresholds for the dual of quasicrystals have also been estimated in Refs. (811). Those of the dual of kagomé, named the dice lattice are reported in Ref. (8). The duals of periodic 3-dimensional $f c c, h c p$ and diamond lattices have been estimated very recently [5].

We also include in the sample the case of rigidity percolation [13]. The bond percolation threshold for the existence of stress carrying paths have been recently determined [14] in a lattice generated by randomly displacing the sites of a triangular lattice in dimension $d=2$.

\section{B. Site versus bond percolation thresholds}

We can easily eliminate $q$ between the expressions of $p_{c s}$ and $p_{c b}$ using Eqs. (1) and (2). We actually get the following invariant which combines both percolation thresholds with the dimension,

$$
\left\{p_{c s}\right\}^{\frac{1}{a_{s}}}\left\{p_{c b}\right\}^{-\frac{1}{a_{b}}}=\frac{\delta}{d}
$$

where $\delta \equiv\left\{p_{o s}\right\}^{\frac{1}{a_{s}}}\left\{p_{o b}\right\}^{-\frac{1}{a_{b}}}$,

Our above formula shows for the first time a topology invariance with 
respect to percolation thresholds. To check its validity against our sample of lattices, it is more convenient to rewrite it as

$$
p_{c s}=\delta^{a_{s}}\left\{d^{-a_{b}} p_{c b}\right\}^{\frac{a_{s}}{a_{b}}}
$$

to have a better graphic representation.

We have plotted in Fig. (1) $\log \left(p_{c s}\right)$ vs $\log \left(d^{-a_{b}} p_{c b}\right)$. After Eq. (3), the universal curve in Fig. (1) reduces to a straigt line. The agreement of Eq. (3) with the data is impressive for all the lattices, with the exception of the dual of diamond, which is far from the straight line in Fig. (1).

Percolation thresholds decrease with increasing space dimensionality. Therefore, the best test for the law when $d$ varies from 2 to 6 is provided by the relative deviation $\Delta p_{c} / p_{c}$, since the absolute deviation $\Delta p_{c}$ is necessarily small at large $d$. That is why we have plotted in Fig. (1) the logarithm of the quantities of interest, in linear scale, instead of the quantities in a logathmic scale. The deviation from the straight line in Fig. (1) is thus a measure of the relative deviation from the law. Also, the accuracy of Eq. (2) is evident from the correlation coefficient,

$$
r=\frac{n \sum x y-\sum x \sum y}{\sqrt{\left[n \sum x^{2}-\left(\sum x\right)^{2}\right]\left[\left(n \sum y^{2}-\left(\sum y\right)^{2}\right]\right.}}
$$

on the data $(x, y)$ on the $n=9$ lattices of the first class and the $n=13$ lattices of the second class (once the dual of diamond has been excluded) in a linear regression analysis. The result is $r=0.997$ for the first class, and $r=0.9994$ for the second class. Yet the smaller value of $r$ in the first class may be due to the lack of accuracy in the determination of the percolation thresholds for the octa- and dodecagonal quasicrystals and their duals. The associated numerical estimate is reported with only two decimals for bonds, and three decimals for sites [10], against four decimals in most other cases.

The parameters $p_{0 s}, p_{0 b}, a_{s}$ and $a_{b}$ have been determined from the fit of Eqs (1) and (2) [3]. However, these equations are only approximate, so that the values of $a_{s}$ and $\delta$ deduced from this work is not the best choice for these parameters entering Eq. (3) or Eq. (4). Instead, we have determined $a_{s}$ and $\delta$ independently, from the least square fit of the data in Fig. (1) by a 
straight line. The same linear regression analysis which has provided us with the correlation coefficient $r$ above mentioned gives

$$
\left\{\begin{array}{l}
a_{s}=0.3670 ; \delta=1.3638 \text { for the first class } \\
a_{s}=0.6068 ; \delta=1.9340 \text { for the second class }
\end{array}\right.
$$

We have listed in Table (1) the quantity

$$
C=\frac{\delta}{d\left(p_{c s}^{\frac{1}{a_{s}}} p_{c b}^{-\frac{1}{a_{b}}}\right)}
$$

The relative error with respect to Eq. (3) for each lattice is then $|C-1|$.

Though the deviations are small, they are significant, showing our formula is not exact. One consequence is the inconsistency in the numerical value of $a_{s}$ in Eq. (6), different from the value of $a_{s}$ deduced from the least-square fit of Eq. (1) in ref. [3] in the case of the second class. In particular, we have checked that this difference is not solely related to the addition of many lattices to the initial sample in [3] for both classes: when all the lattices in the present work are taken into account, $a_{s}$ deduced from the fit of Eq. (1) is 0.618 for the second class, close to 0.616 reported when the samples are reduced to the initial set of basic lattices [3]. The difference with 0.6068 in Eq. (6) is then due to the fact that none of the Eqs $(1,2)$, and (4) are exact.

Actually, from Eqs. (7), an upper limit of the relative error is given by

$$
|C-1| \leq \frac{1}{a_{s}} \frac{\Delta p_{c s}}{p_{c s}}+\frac{1}{a_{b}} \frac{\Delta p_{c b}}{p_{c b}} .
$$

It is reached when deviations of $p_{c s}$ and $p_{c b}$ from Eqs $(1,2)$ are not correlated. For the second class, as an example, the relative accuracy of Eq.(1) according to reference [3] is $\Delta p_{c s} / p_{c s}=3 \%$, while that of Eq. (2) is $\Delta p_{c b} / p_{c b}=2 \%$, hence $|C-1| \leq 7 \%$. According to Table 1 , the deviation of $C$ from unity is better than expected, namely within 5 per cent. This accuracy for the universal law in Eq. (3) means a correlation between deviations of Eqs (1) and (2), so that Eq. (3) can be satisfied, despite Eqs. (1) and (2) are not. The most outstanding illustration is provided by the rigidity percolation case. Here, Eq. 
(3) is satisfied, with $C=0.97$, but the site and the bond percolation thresholds according to Eqs. (1) and (2) are those expected for a lattice with $q=3$, while the actual coordination number is $q=6$.

The topology invariance law in Eq. (3) is satisfied (within 5\%) for all the lattices with the exception of the dual of diamond. We then conclude that the dimension $d$ which is the only variable in this equation is a robust parameter. On another hand, the percolation thresholds of some lattices are significantly different from those expected from Eqs. (1) and (2). In a prior work [15], we had already given arguments to substitute $q$ by $(q-1)$ in formulas to determine percolation thresholds in frustrated lattices. The rigidity percolation with $q_{e f f}=3$ against $q=6$ is another evidence that the coordination number is not a robust parameter. This is a limit to the application of Eqs (1) and (2) as long as any model to determine $q_{e f f}$ [3] from the topology of the lattice is lacking. Eq. (3) is then a significant improvement, as it does not involve any additional unknown parameter. Moreover, this law is the first link between site and bond percolation thresholds.

We have already argued [3] that Eqs. $(1,2)$ violate the $(q-1)^{-1}$ expansions for the d-dimensional simple hypercubic lattice percolation thresholds, and do not match the Bethe asymptotic limit. This is also the case for Eq. (3). Actually, in the large $d$ limit, the leading term for both percolation thresholds should be the Bethe term $p_{c}^{s} \sim p_{c}^{b} \sim 1 /(q-1) \sim 1 /(2 d)$. After Eq. (4) this limit requires:

$$
\frac{1}{a_{s}}-\frac{1}{a_{b}}=1 ; \delta=\frac{1}{2}
$$

The deviation from our results in Eq. (6) gives evidence that our formula in Eqs. $(3,6)$ is not compatible with exact $1 / \mathrm{d}$ expansion. We then expect that the range of validity for our farmula is the same as for Eqs $(1,2)$, namely $d \leq 7$, after Ref. 3 .

\section{Conclusion}

Lattices with a higher coordination number have lower percolation thresholds [5]. Yet there are exceptions like the Kagomé lattice at $d=2$ and the dual of diamond at $d=3$.

At $d=2$ there are theoretical arguments according to which the bond 
percolation threshold of a lattice and its dual should add to one. However, no regularity has been found in three dimensions [5].

The universal law provided by Eq. (3) is then the first relation which links site and bond percolation thresholds. It applies in any dimension up to $d=6$, and extends to any kind of lattice. Departure from this universal law for all the lattices in any dimension is within few per cent.

In addition, the robustness of our universal law supports the extension to more complex problems such as rigidity percolation. We then expect it to be satisfied for any percolation problem, with very few out-liers. The only one we have found so far is the dual of diamond for which indeed an anomalous site percolation threshold has been reported.

\section{Acknowledgments.}

We would like to thank Monsieur Dietrich Stauffer for stimulating vibrations. 


\section{References}

1. M. Sahimi, Application of Percolation Theory (Taylor and Francis, London, 1994).

2. D. Stauffer and A. Aharony, Introduction to Percolation Theory, 2nd ed. (Taylor and Francis, London, 1994).

3. S. Galam and A. Mauger, Phys. Rev. E $\underline{53}, 2177$ (1996).

4. Van der Marck, Phys. Rev. E $\underline{55}, 1228$ (1997); S. Galam and A. Mauger, Phys. Rev. E $\underline{55}, 1230$ (1997).

5. Van der Marck, Phys. Rev. E in press.

6. S. Galam and A. Mauger, Phys. Rev. E, in press.

7. V.K.S. Shante and S. Kirpatrick, Adv. Phys. 20, 326 (1971).

8. J. P. Lu and J. L. Birman, J. Stat. Phys. $\underline{40}, 1057$ (1987).

9. F. Babalievski and O. Pesshev, C. R. Acad. Sci. Bulg. 411, 85 (1988).

10. F. Yonesava, S. Sakamoto, K. Aoki, S. Nose and M. Hori, J. Non-Cryst. Solids $\underline{123}, 73$ (1988).

11. F. Babalievski, Physica A220, 245 (1995).

12. D. Leduie and J. Teillet, J. Non-Cryst. Solids 191, 216 (1995).

13. C. Moukarzel and P.M. Duxbury, Phys. Rev. Lett., in press.

14. D. Jacobs and M. F. Thorpe, Phys. Rev. Lett., in press.

15. S. Galam and A. Mauger, Physica A205, 502 (1994). 


\section{Figure captions}

Fig. 1. Decimal logarithm of site versus bond percolation thresholds. 


\begin{tabular}{|c|c|c|c|}
\hline \multicolumn{4}{|c|}{ first class } \\
\hline Lattice & $p_{c}($ site $)$ & $p_{c}$ (bond) & $C$ \\
\hline Square & 0.5928 & 0.5 & 1.05 \\
Honeycomb & 0.6962 & 0.6527 & 0.99 \\
Triangular & 0.5 & 0.34729 & 0.98 \\
Rigid perco. & 0.6975 & 0.644 & 0.97 \\
Dice & 0.5851 & 0.476 & 1.01 \\
Penrose & 0.5837 & 0.477 & 1.02 \\
Octa.chem.links & 0.585 & 0.48 & 1.01 \\
Octa.ferro.links & 0.543 & 0.40 & 0.98 \\
Dode.chem.links & 0.628 & 0.54 & 1.00 \\
\hline \hline \multicolumn{4}{|c|}{ second class } \\
\hline Lattice & $p_{c}(\mathrm{site})$ & $p_{c}$ (bond) & $C$ \\
\hline Kagomé & 0.6527 & 0.5244 & 0.99 \\
dual of Penrose & 0.6381 & 0.5233 & 1.02 \\
Dode.ferro.links & 0.617 & 0.495 & 1.02 \\
hexag. compact & 0.204 & 0.124 & 0.96 \\
stacked triangle & 0.2623 & 0.1859 & 0.98 \\
Diamond & 0.43 & 0.1859 & 0.95 \\
simple cubic & 0.3116 & 0.2488 & 1.00 \\
bcc & 0.246 & 0.1803 & 1.05 \\
fcc & 0.198 & 0.119 & 0.96 \\
dual of fcc & 0.3341 & 0.2703 & 0.98 \\
dual hexag. comp. & 0.3101 & 0.2573 & 1.05 \\
dual of diamond & 0.3904 & 0.235 & 0.66 \\
sc (d=4) & 0.197 & 0.1601 & 1.00 \\
sc (d=5) & 0.141 & 0.1182 & 1.00 \\
sc (d=6) & 0.107 & 0.0942 & 1.03 \\
\hline
\end{tabular}

Table 1: exact estimates of percolation thresholds $p_{c}$ and universal constant $C$ defined in Eq. (6). Deviation of $C$ from unity measures the departure from the invariant. All the lattices belonging to the first class are in dimension $d=2$. Those of the second class are in $d=2$ (the three first ones), $d=3$ (next nine lattices) and $d=4,5,6$ for the simple hypercube (sc). 